\title{
The Bogolubov representation of the polaron model and its completely integrable RPA-approximation
}

\author{
N.N. Bogolubov (jr. ${ }^{112} ;$, Ya.A. Prykarpatsky ${ }^{314}$; A.A. Ghazaryan ${ }^{5}$ \\ ${ }^{1}$ Steklov Mathematical Institute of the Russian Academy of Sciences, Moscow, Russian Federation \\ 2 Abdus Salam International Centre for Theoretical Physics, Trieste, Italy \\ 3 Institute of Mathematics, Pedagogical University of Cracow, Poland \\ ${ }^{4}$ Drohobych Ivan Franko State Pedagogical University, Lviv region, Ukraine \\ 5 The Lomonosov Moscow State University, Department of Physics, Moscow, Russian Federation
}

Received November 13, 2009, in final form March 25, 2010

\begin{abstract}
The polaron model in ionic crystal is studied in the Bogolubov representation using a special RPA-approximation. A new exactly solvable approximated polaron model is derived and described in detail. Its free energy at finite temperature is calculated analytically. The polaron free energy in the constant magnetic field at finite temperature is also discussed. Based on the structure of the Bogolubov unitary transformed polaron Hamiltonian there is stated a very important new result: the full polaron model is exactly solvable.
\end{abstract}

Key words: polaron, polaron model, constant magnetic field, RPA-approximation

PACS: $71.38 .+i$

\section{Introduction}

The polaron concepts, which was first introduced by Landau [2], is one of the main pillars which the theoretical analysis of materials with strong electron-phonon coupling rests on. In these compounds, the coupling between the electron and the lattice leads to a lattice deformation whose potential tends to bind the electron to the deformed region of the crystal. This process, which has been called self-trapping because of the potential depending on the state of the electron, does not destroy translational invariance, even if the lattice deformation is confined to a single lattice site (small polaron) [1, 5, 7, 9]. Quantum mechanical tunneling between different lattice sites restores this symmetry and ensures that a self-trapped electron forms [10, 11] an itinerant polaronic quasiparticle.

A polaron is a quasiparticle composed of a charge and its accompanying polarization field. A slowly moving electron in a dielectric crystal, interacting with lattice ions through long-range forces will be permanently surrounded by a region of lattice polarization and deformation caused by the moving electron. Moving through the crystal, the electron carries the lattice distortion along with it. Thus, one may speak of a cloud of phonons accompanying the electron.

The resulting lattice polarization acts as a potential wall that hinders the movements of the charge and thus decreases its mobility. Polarons have a spin, though two close-by polarons are spinless. The latter is called a bipolaron. In materials science and chemistry, a polaron is formed when a charge within a molecular chain effects the local nuclear geometry, causing an attenuation (or even reversal) of the nearby bond alternation amplitudes. This "excited state" possesses an energy level between the lower and upper bands.

As it is well known [1, 13, 14], the quantum model of the polaron in the ion crystal of volume

\footnotetext{
*E-mail: nikolai_bogolubov@hotmail.com

†E-mail: yarpry@gmail.com
} 
$\Lambda \subset \mathbb{E}^{3}$ can be described [1] by means of the Hamiltonian operator

$$
\hat{H}_{\mathrm{p}}=\frac{\hat{p}^{2}}{2 m} \otimes \mathbf{1}+\sum_{(f)} \mathbf{1} \otimes\left(b_{f}^{+} b_{f}+1 / 2\right) \hbar \omega_{f}+\frac{1}{\Lambda^{1 / 2}} \sum_{(f)} L_{f}\left(\frac{\hbar}{2 \omega_{f}}\right)^{1 / 2} \mathrm{e}^{i\langle f, r\rangle} \mathbf{1} \otimes\left(b_{f}+b_{-f}^{+}\right),
$$

acting in the Hilbert space $L_{2}(\Lambda ; \mathbb{C}) \otimes \Phi(\Lambda ; \mathbb{C})$, where $\Phi(\Lambda ; \mathbb{C})$ is the corresponding Fock space for the phonon quasi-particle states in the crystal, $m$ is an effective electron mass, $\hat{p}:=\frac{\hbar}{i} \nabla$ is its momentum operator, $b_{f}^{+}$and $b_{f}, f \in 2 \pi \Lambda^{-1 / 3} \mathbb{Z}^{3}$, are, respectively, Bose-operators of creation and annihilation of phonons with energy $\hbar \omega_{f} \in \mathbb{R}_{+}$, the coefficient $L_{f}=\sqrt{\alpha}|f|^{-1}, \alpha \in \mathbb{R}_{+}$, is an intensity parameter of the polaron bond in the crystal and $\langle.,$.$\rangle is the ordinary scalar product in$ the Euclidean space $\mathbb{E}^{3}$.

As it was also shown in [1, 4], Hamiltonian (1) can be transformed by means of the unitary transformation

$$
U:=\exp \left(\mathrm{i} \sum_{(f)}\langle f, r\rangle \mathbf{1} \otimes b_{f}^{+} b_{f}\right)
$$

into the following form:

$$
\hat{\mathcal{H}}_{\mathrm{p}}=\frac{1}{2 m}\left(\hat{p} \otimes \mathbf{1}-\mathbf{1} \otimes \sum_{(f)} \hbar f b_{f}^{+} b_{f}\right)^{2}+\sum_{(f)} \hbar \omega_{f} \mathbf{1} \otimes b_{f}^{+} b_{f}+\frac{1}{\Lambda^{1 / 2}} \sum_{(f)} L_{f}\left(\frac{\hbar}{2 \omega_{f}}\right)^{1 / 2} \mathbf{1} \otimes\left(b_{f}+b_{-f}^{+}\right),
$$

where it is clearly seen a quantum nature of the polaron structure, which does not depend on the force of the interaction parameter $L_{f}$. This conclusion can be also made from the fact that model (11) possesses a conservation law of the general electron-phonon momentum:

$$
\hat{P}=\hat{p} \otimes \mathbf{1}+\mathbf{1} \otimes \sum_{(f)} \hbar f b_{f}^{+} b_{f},
$$

that is $\left[\hat{\mathcal{H}}_{\mathrm{p}}, \hat{P}\right]=0$. It is interesting to note, that the analytical studies of statistical properties of the model of polaron in all of the works on this problem [1, 3, 6, 13, 21], except the works [4, 5], were based on the Hamiltonian expression (11). But, as it was pointed out in [1], the statistical properties of the model do not depend on the unitary-equivalent representation choice for operator (11). In particular, expression (3) can be easily rewritten equivalently, making use of the normal operator ordering, as

$$
\begin{aligned}
\hat{\mathcal{H}}_{\mathrm{p}}= & \frac{1}{2 m}\left(\hat{p} \otimes \mathbf{1}-\mathbf{1} \otimes \sum_{(f)} \hbar f b_{f}^{+} b_{f}\right)^{2}+\sum_{(f)} \hbar \omega_{f} \mathbf{1} \otimes b_{f}^{+} b_{f}+\frac{1}{\Lambda^{1 / 2}} \sum_{(f)} L_{f}\left(\frac{\hbar}{2 \omega_{f}}\right) 1 / \mathbf{1} \otimes\left(b_{f}+b_{-f}^{+}\right) \\
= & \frac{1}{2 m} \hat{p}^{2} \otimes \mathbf{1}-\frac{1}{m} \sum_{(f, g)}\left\langle\hat{p}_{g}, \hbar f\right\rangle \otimes b_{g}^{+} b_{g} b_{f}^{+} b_{f}+\frac{1}{2 m} \sum_{(f, g)}\langle\hbar f, \hbar g\rangle \mathbf{1} \otimes b_{f}^{+} b_{f} b_{g}^{+} b_{g} \\
& +\sum_{(f)} \hbar \omega_{f} \mathbf{1} \otimes b_{f}^{+} b_{f}+\frac{1}{\Lambda^{1 / 2}} \sum_{(f)} L_{f}\left(\frac{\hbar}{2 \omega_{f}}\right)^{1 / 2} \mathbf{1} \otimes\left(b_{f}+b_{-f}^{+}\right) \\
= & \frac{1}{2 m} \hat{p}^{2} \otimes \mathbf{1}-\sum_{(f)} \frac{1}{2 m} \hat{p}_{f}^{2} \otimes b_{f}^{+} b_{f}+\sum_{(f)}\left[\left(\hat{p}_{f}-\hbar f\right)^{2}+\hbar \omega_{f}\right] \otimes b_{f}^{+} b_{f} \\
& +\frac{1}{2 m} \sum_{(f, g)}\langle\hbar f, \hbar g\rangle \mathbf{1} \otimes b_{f}^{+} b_{g}^{+} b_{g} b_{f}-\frac{1}{m} \sum_{(f, g)}\left\langle\hat{p}_{f}, \hbar g\right\rangle \otimes b_{f}^{+} b_{g}^{+} b_{g} b_{f} \\
& +\frac{1}{\Lambda^{1 / 2}} \sum_{(f)} L_{f}\left(\frac{\hbar}{2 \omega_{f}}\right)^{1 / 2} \mathbf{1} \otimes\left(b_{f}+b_{-f}^{+}\right) \\
= & \frac{1}{2 m} \hat{p}^{2} \otimes \mathbf{1}-\sum_{(f)} \frac{1}{2 m} \hat{p}_{f}^{2} \otimes b_{f}^{+} b_{f}+\frac{1}{2 m} \sum_{(f)}\left[\left(\hat{p}_{f}-\hbar f\right)^{2}+\hbar \omega_{f} \mathbf{1}\right] \otimes b_{f}^{+} b_{f}
\end{aligned}
$$




$$
\begin{aligned}
& -\frac{1}{2 m} \sum_{(f, g)}\left(\left\langle\hat{p}_{f}, \hbar g\right\rangle+\left\langle\hat{p}_{g}, \hbar f\right\rangle\right) \otimes b_{f}^{+} b_{g}^{+} b_{g} b_{f} \\
& +\frac{1}{2 m} \sum_{(f, g)}\langle\hbar f, \hbar g\rangle \mathbf{1} \otimes b_{f}^{+} b_{g}^{+} b_{g} b_{f}+\frac{1}{\Lambda^{1 / 2}} \sum_{(f)} L_{f}\left(\frac{\hbar}{2 \omega_{f}}\right)^{1 / 2} \mathbf{1} \otimes\left(b_{f}+b_{-f}^{+}\right),
\end{aligned}
$$

where we made use of the tensor operator representation $\hat{p} \otimes \mathbf{1}=\sum_{(f)} \hat{p}_{f} \otimes b_{f}^{+} b_{f}=\sum_{(f)} \hat{p} \hat{\mathbf{N}}^{-1} \otimes$ $b_{f}^{+} b_{f}, \quad \hat{\mathbf{N}}:=\sum_{(f)} b_{f}^{+} b_{f}$, in the Fock space $\Phi(\Lambda ; \mathbb{C})$ and of the related commutation properties: $\left[\hat{p}_{f}, \hat{p}_{g}\right]=0=\left[\hat{p}_{g}, b_{f}^{+} b_{f}\right]$ for all $f, g \in 2 \pi \Lambda^{-1 / 3} \mathbb{Z}^{3}$. We need to mention here that this representation of the Hamiltonian operator (3) possesses a natural physical interpretation as a polaron model with collectively separated interaction potentials. This fact proves to have become very important in our analysis that follows below.

In a series of papers on the polaron theory [1, [6] the oscillator approximation of the Hamiltonian (1) was analyzed. This approximation leads to the so-called "linearized" model of the polaron, when only the first member of the expansion $\exp (\mathrm{i}\langle r, f\rangle) \simeq 1+\mathrm{i}\langle r, f\rangle$ in (1) was taken into account and the quadratic compensating part $K_{0} r^{2} / 2$, where $K_{0}^{2}=\frac{1}{3 \Lambda} \sum_{(f)} L_{f}^{2} f^{2} \omega_{f}^{-2}$, was added into the initial Hamiltonian. Under these conditions, the resulting Hamiltonian persists to be translationalinvariant which makes it possible to analyze its thermodynamic properties as $N \rightarrow \infty, \Lambda \rightarrow \infty$.

Concerning the Hamiltonian representation (5) one can make the following very important observation: the operator term $\hat{\mathcal{V}}_{\mathrm{p}}^{(1)}:=\frac{1}{2 m} \sum_{(f, g)}\left(\left\langle\hat{p}_{f}, \hbar g\right\rangle+\left\langle\hat{p}_{g}, \hbar f\right\rangle+\langle\hbar f, \hbar g\rangle \mathbf{1}\right) \otimes b_{f}^{+} b_{g}^{+} b_{g} b_{f}$, being written in the normally ordered secondly quantized form, gives rise to the following effective twoparticle operator expression in an $N$-particle invariant Fock subspace [14, 16, 17, 19]:

$$
\hat{\mathcal{V}}_{N}^{(1)}=\frac{1}{2 m} \sum_{k \neq j=1}^{N}\left(\frac{1}{N}\left\langle\hat{\mathrm{p}}\left(y_{j}\right) \otimes \hat{\Pi}\left(y_{k}\right)+\hat{\mathrm{p}}\left(y_{k}\right) \otimes \hat{\Pi}\left(y_{j}\right)\right\rangle+\left\langle\hat{\Pi}\left(y_{k}\right), \hat{\Pi}\left(y_{j}\right)\right\rangle\right),
$$

acting in the Hilbert space $L_{2}(\Lambda ; \mathbb{C}) \otimes L_{2, \text { sym }}\left(\Lambda^{N} ; \mathbb{C}\right)$, where we denoted by $\hat{\Pi}(y), y \in \Lambda$, the respectively modified momentum operator of the crystal deformations and $\hat{\mathrm{p}}(y), y \in \Lambda$, the uniformly distributed polaron momentum.

Taking into account the well-known [14, 17, 18] random phase approximation (RPA) for the two-particle phonon excitations in crystal, one can obtain that expression (6) transforms into zero, since $\sum_{k \neq j=1}^{N}\left\langle\hat{\Pi}\left(y_{k}\right), \hat{\Pi}\left(y_{j}\right)\right\rangle=0=\sum_{k \neq j=1}^{N} \frac{1}{N}\left(\left\langle\hat{\mathrm{p}}\left(y_{j}\right) \otimes \hat{\Pi}\left(y_{k}\right)+\hat{\mathrm{p}}\left(y_{k}\right) \otimes \hat{\Pi}\left(y_{j}\right)\right\rangle\right)$ weakly in $L_{2, \operatorname{sym}}\left(\Lambda^{N} ; \mathbb{C}\right)$ owing to the stability of the crystal deformations, generated by the polaron interaction. Since we are interested in the statistical properties of our polaron model, the above discussed RPAapproximation well fits to this aim, because the corresponding statistical sum is calculated as the average value of statistical operator over all Hamiltonian (5) eigenstates. Thus, we can consider a polaron model Hamiltonian within the RPA-approximation in the following reduced form:

$$
\begin{aligned}
\hat{\mathcal{H}}_{\mathrm{p}}^{(0)}= & \frac{1}{2 m} \hat{p}^{2} \otimes\left(\mathbf{1}-\hat{\mathbf{N}}^{-1}\right)+\frac{1}{2 m} \sum_{(f)}\left(\hat{p}_{f}-\hbar f\right)^{2} \otimes b_{f}^{+} b_{f} \\
& +\sum_{(f)} \hbar \omega_{f} \mathbf{1} \otimes b_{f}^{+} b_{f}+\frac{1}{\Lambda^{1 / 2}} \sum_{(f)} L_{f}\left(\frac{\hbar}{2 \omega_{f}}\right)^{1 / 2} \mathbf{1} \otimes\left(b_{f}+b_{-f}^{+}\right),
\end{aligned}
$$

being a well defined operator expression bounded from below in the Hilbert space $L_{2}(\Lambda ; \mathbb{C}) \otimes$ $\Phi(\Lambda ; \mathbb{C})$.

It can be shown using the standard considerations, devised in [6, 20] jointly with the Bogolubov inequality [1], that the corresponding statistical sums for the Hamiltonian operators (77) and (3) in the thermodynamical limit are asymptotically equal as the intensity parameter $\alpha \rightarrow \infty$. The analytical details of this analysis will be presented in the Part 2 of this work. Thereby, we believe that our model (7), describing the polaron properties within the RPA-approximation, is more appropriate in studying its thermodynamics, remaining a priori translation-invariant. We will 
study this model herein below in detail. Moreover, we will investigate this polaron model in the external magnetic field $B=\operatorname{rot} A$, where, by definition, the vector potential $A=\left(0,-m \omega_{\mathrm{c}} x, 0\right)^{\top} \in$ $\mathbb{E}^{3}$ is directed along the axis $O y$ of the Euclidian space $\mathbb{E}^{3}$ and $\omega_{\mathrm{c}} \in \mathbb{R}_{+}$denotes the corresponding "cyclotronic" frequency. In this case Hamiltonian (5) has the form

$$
\begin{aligned}
\hat{\mathcal{H}}_{\mathrm{p}}^{(\mu)}= & \frac{1}{2 m}\left(\hat{p}^{(\mu) 2}+\hat{p}_{z}^{2}\right) \otimes\left(\mathbf{1}-\hat{\mathbf{N}}^{-1}\right)-\sum_{(f)} \frac{1}{2 m}\left(\hat{p}_{f}^{(\mu) 2}+\hat{p}_{f_{z}}^{2}\right) \otimes b_{f}^{+} b_{f} \\
& +\frac{1}{2 m} \sum_{(\bar{f})}\left(\hat{p}_{f}^{(\mu)}-\hbar \bar{f}\right)^{2} \otimes b_{f}^{+} b_{f}+\frac{1}{2 m} \sum_{\left(f_{z}\right)}\left(\hat{p}_{f_{z}}-\hbar f_{z}\right)^{2} \otimes b_{f}^{+} b_{f} \\
& +\sum_{(f)} \hbar \omega_{f} \mathbf{1} \otimes b_{f}^{+} b_{f}+\frac{1}{\Lambda^{1 / 2}} \sum_{(f)} L_{f}\left(\frac{\hbar}{2 \omega_{f}}\right)^{1 / 2} \mathbf{1} \otimes\left(b_{f}+b_{-f}^{+}\right),
\end{aligned}
$$

where by definition $\hat{p}_{f}^{(\mu)}=\left(\hat{p}_{f_{x}}, \hat{p}_{f_{y}}+m \omega_{\mathrm{c}} x\right)^{\top}, \bar{f}=\left(f_{x}, f_{y}\right)^{\top}$, pertaining to the quadratic structure of the phonon operators.

\section{The RPA-approximated polaron model}

To study the thermodynamics of the $R P A$-approximated polaron model (5), we need to calculate the statistical sum

$$
Z_{\mathrm{p}}^{(0)}=\operatorname{Sp} \exp \left(-\beta \hat{\mathcal{H}}_{\mathrm{p}}^{(0)}\right),
$$

where $\beta=1 / k T$ is the inverse temperature in the Boltzmann units. Then, taking into account that

$$
\hat{\mathcal{H}}_{\mathrm{p}}^{(0)}=\frac{1}{2 m} \hat{p}^{2} \otimes\left(\mathbf{1}-\hat{\mathbf{N}}^{-1}\right)+\sum_{(f)} \hbar \hat{\omega}_{f} \otimes b_{f}^{+} b_{f}+\frac{1}{\Lambda^{1 / 2}} \sum_{(f)} L_{f}\left(\frac{\hbar}{2 \omega_{f}}\right)^{1 / 2} \mathbf{1} \otimes\left(b_{f}+b_{-f}^{+}\right)
$$

where the operators

$$
\hat{\omega}_{f}:=\left[\frac{1}{2 m}\left(\hat{p}_{f} / \hbar-f\right)^{2}+\omega_{f}\right], \quad \hat{n}_{f}:=b_{f}^{+} b_{f}
$$

commute to each other, that is $\left[\hat{\omega}_{f}, \hat{n}_{f}\right]=0$, we can calculate expression (9) classically, having reduced it to the form

$$
Z_{\mathrm{p}}^{(0)}=\operatorname{Sp}\left(-\beta \mathcal{H}_{\mathrm{p}}^{(0)}\right)=\operatorname{Sp}_{(e)} Z_{\mathrm{ph}}^{(0)}(\hat{p})
$$

Here, by definition, we denoted the "phonon" part of the statistical sum as

$$
Z_{\mathrm{ph}}^{(0)}(\hat{p})=\operatorname{Sp}_{(\mathrm{ph})} \exp \left(-\beta \hat{\mathcal{H}}_{\mathrm{p}}^{(0)}\right)
$$

Since the operator $\hat{\mathcal{H}}_{\mathrm{ph}}^{(0)}$ is a quadratic form with respect to the phonon operators, it is easy to calculate that the change of variables

$$
b_{f}=\tilde{b}_{f}-c_{f}\left(\hat{p}, \hat{N}_{f}\right), \quad b_{f}^{+}=\tilde{b}_{f}^{+}-c_{f}\left(\hat{p}, \hat{N}_{f}\right), \quad c_{f}\left(\hat{p}, \hat{N}_{f}\right)=\frac{1}{\Lambda^{1 / 2}} \sum_{(f)} \frac{L_{f}}{\hat{\omega}_{f}}\left(\frac{\hbar}{2 \omega_{f}}\right)^{1 / 2}
$$

transforms it to the canonical diagonal form

$$
\hat{\mathcal{H}}_{\mathrm{ph}}^{(0)}=\frac{1}{2 m} \hat{p}^{2} \otimes\left(\mathbf{1}-\hat{\mathbf{N}}^{-1}\right)+\sum_{(f)} \hbar \hat{\omega}_{f} \otimes \tilde{b}_{f}^{+} \tilde{b}_{f}+\frac{1}{\Lambda} \sum_{(f)} \frac{L_{f}^{2}}{2 \hat{\omega}_{f} \omega_{f}} \otimes \mathbf{1}
$$

Here we need to mention that the operator $\hat{\omega}_{f}: L_{2}(\Lambda ; \mathbb{C}) \rightarrow L_{2}(\Lambda ; \mathbb{C})$ is a strongly positive defined expression. We also notice here, as it was done in [1], that the phonon frequencies satisfy the symmetry condition $\omega_{f}=\omega_{-f}$ for all vectors $f \in 2 \pi \Lambda^{-1 / 3} \mathbb{Z}^{3}$. A similar symmetry condition also holds for the operator quantities $\hat{\omega}_{f}$, that is $\hat{\omega}_{f}=\hat{\omega}_{-f}^{+}$for all $f \in 2 \pi \Lambda^{-1 / 3} \mathbb{Z}^{3}$. Based on 
representation (13), it is easy to find the following expression for the phonon part of statistical sum (11):

$$
\begin{aligned}
& Z_{\mathrm{ph}}^{(0)}(\hat{p})=\exp \left(-\frac{\beta}{2 m} \hat{p}^{2}\right) \operatorname{Sp}_{(\mathrm{ph})} \prod_{(f)} \sum_{\left(\tilde{N}_{f}\right)} \exp \left[\frac{\beta \hat{p}^{2}}{2 m\left[\hat{N}_{f}+c_{f}^{2}\left(\hat{p}, \tilde{N}_{f}\right)\right]}-\beta \hbar \tilde{N}_{f} \bar{\omega}_{f}\left(\hat{p}, \tilde{N}_{f}\right)\right. \\
&-\left.\beta \frac{L_{f}^{2}}{2 \Lambda \bar{\omega}_{f}\left(\hat{p}, \tilde{N}_{f}\right) \omega_{f}}\right]=\exp \left(-\frac{\beta}{2 m} \hat{p}^{2}\right) \exp \left\{\sum _ { ( f ) } \sum _ { ( N _ { f } \in \mathbb { Z } _ { + } ) } \operatorname { e x p } \left[\frac{\beta \hat{p}^{2}}{2 m\left[N_{f}+c_{f}^{2}\left(\hat{p}, N_{f}\right)\right]}\right.\right. \\
&\left.\left.-\beta \hbar N_{f} \bar{\omega}_{f}\left(\hat{p}, N_{f}\right)-\beta \frac{L_{f}^{2}}{2 \Lambda \bar{\omega}_{f}\left(\hat{p}, N_{f}\right) \omega_{f}}\right]\right\}:=\exp \left(-\frac{\beta}{2 m} \hat{p}^{2}\right) \exp \left\{-\sum_{(f)} F_{f}(\hat{p} ; \beta)\right\},
\end{aligned}
$$

where we denoted by $\tilde{N}_{f}:=\tilde{b}_{f}^{+} \tilde{b}_{f}, f \in 2 \pi \Lambda^{-1 / 3} \mathbb{Z}^{3}$, the shifted phonon density operators and $\bar{\omega}_{f}\left(\hat{p}, N_{f}\right):=\left\{\left(\frac{1}{2 m}\left(\hat{p} /\left[\left(N_{f}+c_{f}^{2}\left(\hat{p}, N_{f}\right) \hbar\right]-f\right)^{2}+\omega_{f}\right\}, f \in 2 \pi \Lambda^{-1 / 3} \mathbb{Z}^{3}\right.\right.$, the related shifted phonon frequencies. Substituting now (14) in (10), we obtain, as a result, an analytical expression for the complete statistical sum of our approximated polaron model:

$$
Z_{\mathrm{p}}^{(0)}=\operatorname{Sp}_{(e)} \exp \left\{-\frac{\beta}{2 m} \hat{p}^{2}-\sum_{(f)} F_{f}(\hat{p} ; \beta)\right\}=\frac{\Lambda}{(2 \pi)^{3}} \int_{\mathbb{E}^{3}} \mathrm{~d}^{3} k \exp \left\{-\frac{\beta}{2 m} k^{2}-\sum_{(f)} F_{f}(k ; \beta)\right\},
$$

where we used the known trick $[1,13,14]$ of changing the discrete $\operatorname{sum} \sum_{(k)}(\ldots)$ by a corresponding integral:

$$
\sum_{(k)}(\ldots)=\frac{\Lambda}{(2 \pi)^{3}} \int_{\mathbb{E}^{3}}(\ldots) \mathrm{d}^{3} k .
$$

Thus, if we calculate the internal sums $\sum_{(f)}(\ldots)$ of expression (15) for the given values of phonon frequencies $\omega_{f}$ and the interaction parameter $L_{f}$ for all discrete values $f \in 2 \pi \Lambda^{-1 / 3} \mathbb{Z}^{3}$, then the quasi-gaussian integral $\int_{\mathbb{E}^{3}}(\ldots) \mathrm{d}^{3} k$ can be calculated analytically or by means of approximate and asymptotic methods. In particular, assuming that $L_{f}=\alpha^{1 / 2} /|f|$, and $\omega_{f}=\omega_{0}$ for all $f \in$ $2 \pi \Lambda^{-1 / 3} \mathbb{Z}^{3}$, from (15) and (16) it is easy to obtain an explicit expression for the statistical sum of the polaron model (5), which is not going to be dealt with in this paper.

\section{RPA-approximated polaron model in the static magnetic field}

To study RPA-approximated polaron model in the static magnetic field we will use the Hamiltonian operator (8), which persists to be quadratic in the phonon operators. Taking into account that

$$
\begin{aligned}
\hat{\mathcal{H}}_{\mathrm{p}}^{(\mu)}= & \frac{1}{2 m}\left(\hat{p}^{(\mu) 2}+\hat{p}_{z}^{2}\right) \otimes\left(\mathbf{1}-\hat{\mathbf{N}}^{-1}\right)+\frac{1}{2 m} \sum_{(\bar{f})}\left(\hat{p}_{f}^{(\mu)}-\hbar \bar{f}\right)^{2} \otimes b_{f}^{+} b_{f} \\
& +\frac{1}{2 m} \sum_{\left(f_{z}\right)}\left(\hat{p}_{f_{z}}-\hbar f_{z}\right)^{2} \otimes b_{f}^{+} b_{f}+\sum_{(f)} \hbar \omega_{f} \mathbf{1} \otimes b_{f}^{+} b_{f} \\
& +\frac{1}{\Lambda^{1 / 2}} \sum_{(f)} L_{f}\left(\frac{\hbar}{2 \omega_{f}}\right)^{1 / 2} \mathbf{1} \otimes\left(b_{f}+b_{-f}^{+}\right),
\end{aligned}
$$

where the operators

$$
\left(\hat{p}_{f}^{(\mu)}-\hbar \bar{f}\right)^{2}, \quad\left(\hat{p}_{f_{z}}-\hbar f_{z}\right)^{2}, \quad \hat{n}_{f}=b_{f}^{+} b_{f}
$$

commute to each other. Put, by definition,

$$
\hat{\omega}_{f}:=\omega_{f}+\frac{1}{2 m}\left(\hat{p}_{f}^{(\mu)} / \hbar-\bar{f}\right)^{2}+\frac{1}{2 m}\left(\hat{p}_{f_{z}} / \hbar-f_{z}\right)^{2}, \quad \bar{p}_{f}^{(\mu)}=\left(\hat{p}_{f_{x}}, \hat{p}_{f_{y}}+m \omega_{\mathrm{c}} x\right)^{\top},
$$


for $\bar{f}=\left(f_{x}, f_{y}\right)^{\top} \in \mathbb{E}^{2}$, the respectively split statistical sum

$$
Z_{\mathrm{p}}^{(\mu)}=\operatorname{Sp} \exp \left(-\beta \hat{\mathcal{H}}_{\mathrm{p}}^{(\mu)}\right)=\operatorname{Sp}_{(e)}\left[\operatorname{Sp}_{(\mathrm{ph})} \exp \left(-\beta \hat{\mathcal{H}}_{\mathrm{ph}}^{(\mu)}\right)\right]
$$

can be successfully calculated, where

$$
\hat{\mathcal{H}}_{\mathrm{ph}}^{(\mu)}=\frac{1}{2 m}\left(\hat{p}^{(\mu) 2}+\hat{p}_{z}^{2}\right) \otimes\left(\mathbf{1}-\hat{\mathbf{N}}^{-1}\right)+\sum_{(\bar{f})} \hbar \hat{\omega}_{f} \otimes b_{f}^{+} b_{f}+\frac{1}{\Lambda^{1 / 2}} \sum_{(f)} L_{f}\left(\frac{\hbar}{2 \omega_{f}}\right)^{1 / 2} \mathbf{1} \otimes\left(b_{f}+b_{-f}^{+}\right) .
$$

Let us, first, find the statistical sum

$$
Z_{\mathrm{ph}}^{(\mu)}=\mathrm{Sp}_{(\mathrm{ph})} \exp \left(-\beta \hat{\mathcal{H}}_{\mathrm{ph}}^{(\mu)}\right),
$$

for which, using (19) and (21), we obtain

$$
Z_{\mathrm{p}}^{(\mu)}=\operatorname{Sp}_{(e)} Z_{\mathrm{ph}}^{(\mu)} .
$$

Using now the fact that Hamiltonian (18) is quadratic in the phonon variables and making transformation (12), we obtain

$$
\hat{\mathcal{H}}_{\mathrm{ph}}^{(\mu)}=\frac{1}{2 m}\left(\hat{p}^{(\mu) 2}+\hat{p}_{z}^{2}\right) \otimes\left(\mathbf{1}-\hat{\mathbf{N}}^{-1}\right)+\sum_{(f)} \hbar \hat{\omega}_{f} \tilde{b}_{f}^{+} \tilde{b}_{f}+\frac{1}{\Lambda} \sum_{(f)} \frac{L_{f}^{2}}{2 \hat{\omega}_{f} \omega_{f}} .
$$

Thus, from (23) and (21), one obtains that

$$
\begin{aligned}
Z_{\mathrm{p}}^{(\mu)}= & \exp \left[-\frac{\beta}{2 m}\left(\hat{p}^{(\mu) 2}+\hat{p}_{z}^{2}\right)\right] \operatorname{Sp}_{(\mathrm{ph})} \prod_{(f)} \sum_{\left(\hat{N}_{f}\right)} \exp \left[\frac{\beta\left(\hat{p}^{(\mu) 2}+\hat{p}_{z}^{2}\right)}{2 m\left[\tilde{N}_{f}+c_{f}^{2}\left(\hat{p}^{(\mu)}, k_{z} ; \tilde{N}_{f}\right)\right]}\right. \\
& \left.-\beta \hbar \hat{N}_{f} \bar{\omega}_{f}\left(\hat{p}^{(\mu)}, \hat{p}_{z} ; \hat{N}_{f}\right)-\frac{\beta L_{f}^{2}}{2 \Lambda \bar{\omega}_{f}\left(\hat{p}^{(\mu)}, \hat{p}_{z} ; \hat{N}_{f}\right) \omega_{f}}\right] \\
= & \exp \left[-\frac{\beta}{2 m}\left(\hat{p}^{(\mu) 2}+\hat{p}_{z}^{2}\right)\right] \exp \left\{\sum _ { ( f ) } \sum _ { ( N _ { f } \in \mathbb { Z } _ { + } ) } \operatorname { e x p } \left[\frac{\beta\left(\hat{p}^{(\mu) 2}+\hat{p}_{z}^{2}\right)}{2 m\left[\tilde{N}_{f}+c_{f}^{2}\left(\hat{p}^{(\mu)}, k_{z} ; \tilde{N}_{f}\right)\right]}\right.\right. \\
& \left.\left.-\beta \hbar N_{f} \bar{\omega}_{f}\left(\hat{p}^{(\mu)}, \hat{p}_{z} ; N_{f}\right)+\frac{\beta L_{f}^{2}}{2 \Lambda \bar{\omega}_{f}\left(\hat{p}^{(\mu)}, \hat{p}_{z} ; N_{f}\right) \omega_{f}}\right]\right\} \\
:= & \exp \left\{-\frac{\beta}{2 m}\left(\hat{p}^{(\mu) 2}+\hat{p}_{z}^{2}\right)-\sum_{(f)} F_{f}\left(\hat{p}^{(\mu)}, \hat{p}_{z} ; \beta\right)\right\}
\end{aligned}
$$

where $\bar{\omega}_{f}\left(\hat{p}^{(\mu)}, k_{z} ; \beta\right)=\omega_{f}+\frac{1}{2 m}\left(\hat{p}^{(\mu)} /\left[\hbar\left(N_{f}+c_{f}^{2}\right)\right]-\bar{f}\right)^{2}+\frac{1}{2 m}\left(k_{z}-f_{z}\right)^{2}$. Substituting (24) into (22), we obtain an expression for the statistical sum of RPA-approximated polaron model in the magnetic field:

$$
Z_{\mathrm{p}}^{(\mu)}=\operatorname{Sp}_{(e)} \exp \left\{-\frac{\beta}{2 m}\left(\hat{p}^{(\mu) 2}+\hat{p}_{z}^{2}\right)-\sum_{(f)} F_{f}\left(\hat{p}^{(\mu)}, \hat{p}_{z} ; \beta\right)\right\}=\operatorname{Sp}_{(e)} \exp \left(-\beta \overline{\mathcal{H}}_{e}^{(\mu)}\right) .
$$

Here, by definition, we have put

$$
\overline{\mathcal{H}}_{e}^{(\mu)}(\beta):=\frac{1}{2 m} \hat{p}^{(\mu) 2}+\bar{V}_{e}^{(\mu)}\left(\hat{p}^{(\mu)} ; \beta\right)
$$


as an effective polaron Hamiltonian with the operator potential, defined by the expression

$$
\exp \left[-\beta \bar{V}_{e}^{(\mu)}\left(\hat{p}^{(\mu)} ; \beta\right)\right]=\frac{\Lambda^{1 / 3}}{2 \pi} \int_{\mathbb{R}} \mathrm{d} k_{z} \exp \left\{-\sum_{(f)} F_{f}\left(\hat{p}^{(\mu)}, k_{z} ; \beta\right)\right\} .
$$

Thus, the statistical sum (25) is determined completely by means of the spectrum of the one-particle two-dimensional self-adjoint problem

$$
\overline{\mathcal{H}}_{e}^{(\mu)}(\beta) \psi_{n}=\varepsilon_{n}(\beta) \psi_{n},
$$

where the eigenvalues $\varepsilon_{n}(\beta) \in \mathbb{R}_{+}, n \in \mathbb{Z}_{+}$, compile the corresponding modified Landau's spectrum 12 14] and $\psi_{n} \in L_{\infty}\left(\mathbb{E}^{2} ; \mathbb{C}\right), n \in \mathbb{Z}_{+}$, are eigenfunctions of a suitable two-dimensional operator with periodic boundary conditions:

$$
\psi_{n}(x, y)=\psi_{n}\left(x+l_{x} \Lambda^{-1 / 3}, y+l_{y} \Lambda^{-1 / 3}\right)
$$

for all $\left(l_{x}, l_{y}\right)^{\top} \in \mathbb{Z}^{2},(x, y)^{\top} \in \mathbb{E}^{2}$. Then from (25) and (27) it is easy to obtain that

$$
Z_{\mathrm{p}}^{(\mu)}=\sum_{n \in \mathbb{Z}_{+}} \exp \left[-\beta \varepsilon_{n}(\beta)\right],
$$

where the quantities of the Landau spectrum $\varepsilon_{n}(\beta) \in \mathbb{R}, n \in \mathbb{Z}_{+}$, can be found by means of appropriate quantum-mechanical methods, being already another problem to deal with. The energy of the ground state for the reduced polaron model at zero temperature (as $\beta \rightarrow \infty)$ can be calculated [1, 13], as the limit $E_{0}=-\lim _{\beta \rightarrow \infty} \ln Z_{\mathrm{p}}^{(\mu)}$, being also a very interesting and important problem.

\section{The polaron mass}

It is important to mention that the description of our polaron system by means of the Bogolubov canonical transformation (2) gives rise to a direct possibility of calculating the polaron mass in magnetic field within our RPA-approximation both at zero and non-zero temperatures. Namely, based on the considerations of [24], one can consider at zero temperature the least energy state $\left|p^{(\mu)}\right\rangle \in L_{2}(\Lambda ; \mathbb{C}) \otimes \Phi(\Lambda ; \mathbb{C})$ at a small fixed momentum $p^{(\mu)} \in \mathbb{E}^{2}$, if the interaction constant $L_{f}=$ $\sqrt{\alpha}|f|^{-1}, f \in 2 \pi \Lambda^{-1 / 3} \mathbb{Z}^{3}$, and $\alpha \in \mathbb{R}_{+}$is the corresponding dimensionless intensity parameter. Then the polaron energy could be defined as

$$
E\left(\alpha ; p^{(\mu)}\right)=E_{0}(\alpha)+\frac{\left|p^{(\mu)}\right|^{2}}{2 m^{*}(\alpha)}+o\left(\left|p^{(\mu)}\right|^{2}\right),
$$

where $E_{0}(\alpha)$ satisfies the eigenvalue equation

$$
\hat{\mathcal{H}}_{\mathrm{p}}^{(\mu)}\left|p^{(\mu)}\right\rangle=E\left(\alpha ; p^{(\mu)}\right)\left|p^{(\mu)}\right\rangle
$$

under the conditions $\hat{p}^{(\mu)}\left|p^{(\mu)}\right\rangle=p^{(\mu)}\left|p^{(\mu)}\right\rangle$ for a small vector $p^{(\mu)} \in \mathbb{E}^{2}$.

In the case of a non-zero temperature $T>0$ the polaron free energy is determined by means of the following expression:

$$
F(\alpha ; \beta)=-\beta^{-1} \ln \left(Z_{\mathrm{p}}^{(\mu)} / Z_{\mathrm{ph}}^{(\mathrm{can})}\right),
$$

where, by definition, $Z_{\mathrm{ph}}^{(\mathrm{can})}=\mathrm{Sp}_{(\mathrm{ph})} \exp \left(-\beta \hat{\mathcal{H}}_{\mathrm{ph}}^{(\mathrm{can})}\right)$ is the pure statistical sum of the phonon field, and $\hat{\mathcal{H}}_{\mathrm{ph}}^{(\mathrm{can})}=\sum_{(f)} \mathbf{1} \otimes\left(b_{f}^{+} b_{f}+1 / 2\right) \hbar \omega_{f}, \beta=1 / k T$. Making use of the result (31) obtained above one can calculate the average polaron energy as

$$
U(\alpha ; \beta)=\frac{\partial}{\partial \beta}[\beta F(\alpha ; \beta)],
$$


and the proper polaron energy as

$$
E(\alpha ; \beta)=U(\alpha ; \beta)-3 /(2 \beta)
$$

At zero temperature one has:

$$
E(\alpha)=\lim _{\beta \rightarrow \infty} E(\alpha ; \beta)
$$

\section{Conclusion}

The above described one-particle RPA-approximated polaron model in ion crystal can be applied [14], in particular, for analyzing the results of de Haas-van Alfven type experiments. We would also like to mention a polaron gas model, having many interesting applications, which can be similarly investigated by means of powerful methods of the many-particle quantum theory [1, 13, 14, 22], in particular, by means of the methods of quantum current algebra [15] and its representations, as it was recently demonstrated in [20]. Concerning our polaron model and the used RPA-approximation, the following is important to mention: the Hamiltonian operator resulting from the canonical Bogolubov transformation (2) was presented in the canonical normal ordered form as a sum of an exactly solvable operator part and of a part responsible for the manyparticle correlation interaction. The latter appeared to be of the exactly RPA-approximated form, owing to which we could neglect it. This approximation can be used whether the crystal temperature is high enough or the intensity parameter $\alpha \in \mathbb{R}_{+}$is not too small, being $\alpha>5,8$, when a transition of the polaron from a non-localized state to a self-localized state is realized [23, 24].

As it is well known, the temperature dependence of the effective polaron mass is of great interest for physical applications, in particular, with its relation to the interpretation of experiments of cyclotronic resonance in polar crystals [4 6]. Measurements fulfilled in the crystals CdTe and $\mathrm{AgBr}$ in weak magnetic fields at small cyclotronic frequencies demonstrate clearly enough that the corresponding cyclotronic polaron mass grows with temperature at its low values. Thereby, one can expect that the calculation of the magnetic polaron mass based on our results within the RPAapproximation can explain this effect and determine the bounds of the applied method. Moreover, one can expect that our results will confirm the prediction of the existence [13, 21, 22] of the first kind phase transition of a polaron state from its self-localized state to a free state under the action of a strong enough magnetic field. We plan to discuss these intriguing problems in the forthcoming paper.

\section{Supplement}

The Bogolubov unitary transformed Hamiltonian operator (5) can be formally written down as

$$
\hat{\mathcal{H}}_{\mathrm{p}}:=\hat{\mathcal{H}}_{\mathrm{p}}^{(0)}+\hat{\mathcal{V}}_{\mathrm{p}}^{(1)}
$$

where the operator

$$
\hat{\mathcal{V}}_{\mathrm{p}}^{(1)}:=\frac{1}{2 m} \sum_{(f, g)}\left(\left\langle\hat{p}_{f}, \hbar g\right\rangle+\left\langle\hat{p}_{g}, \hbar f\right\rangle+\langle\hbar f, \hbar g\rangle \mathbf{1}\right) \otimes b_{f}^{+} b_{g}^{+} b_{g} b_{f},
$$

is respective for the potential energy of crystal deformations caused by a polaron motion. The following proposition is crucial for our further analysis of statistical properties of the polaron model in the Bogolubov representation (33).

Proposition 6.1 The RPA-approximated polaron operator $\hat{\mathcal{H}}_{\mathrm{p}}^{(0)}: L_{2}(\Lambda ; \mathbb{C}) \otimes \Phi(\Lambda ; \mathbb{C})$ and the crystal deformation energy operator $\hat{\mathcal{V}}_{\mathrm{p}}^{(1)}: L_{2}(\Lambda ; \mathbb{C}) \otimes \Phi(\Lambda ; \mathbb{C})$ are commuting to each other, that is

$$
\left[\hat{\mathcal{H}}_{\mathrm{p}}^{(0)}, \hat{\mathcal{V}}_{\mathrm{p}}^{(1)}\right]=0
$$

identically. 
As a corollary from Proposition 6.1, the operators $\hat{\mathcal{H}}_{\mathrm{p}}^{(0)}, \hat{\mathcal{V}}_{\mathrm{p}}^{(1)}: L_{2}(\Lambda ; \mathbb{C}) \otimes \Phi(\Lambda ; \mathbb{C})$, being selfadjoint, possess a common set of eigenstates, being thereby suitably degenerate. The latter can be used for more exact calculations of the full statistical sum

$$
Z_{\mathrm{p}}=\operatorname{Sp} \exp \left[-\beta\left(\hat{\mathcal{H}}_{\mathrm{p}}^{(0)}+\hat{\mathcal{V}}_{\mathrm{p}}^{(1)}\right)\right]
$$

if we take into account the stability condition imposed on the ion crystal deformations. Namely, we can assume that the following compatible selection conditions

$$
\left\langle(k ; \alpha(k))\left|\hat{\mathcal{V}}_{\mathrm{p}}^{(1)}\right|(k ; \alpha(k))\right\rangle=0, \quad \hat{\mathcal{H}}_{\mathrm{p}}^{(0)}|(k ; \alpha(k))\rangle=\alpha(k)|(k ; \alpha(k))\rangle,
$$

hold for all physically permitted polaron eigenstates $|(k ; \alpha(k))\rangle \in L_{2}(\Lambda ; \mathbb{C}) \otimes \Phi(\Lambda ; \mathbb{C})$ of the $\operatorname{RPA}$ approximated polaron operator $\hat{\mathcal{H}}_{\mathrm{p}}^{(0)}: L_{2}(\Lambda ; \mathbb{C}) \otimes \Phi(\Lambda ; \mathbb{C})$. Then the corresponding statistical sum (36) reduces, owing to constraints (37), to

$$
Z_{\mathrm{p}, \text { red }}=\operatorname{Sp}_{(\text {red })}\left(\exp \left(-\beta \hat{\mathcal{H}}_{\mathrm{p}}^{(0)}\right) \exp \left(-\beta \hat{\mathcal{V}}_{\mathrm{p}}^{(1)}\right)\right)=\operatorname{Sp}_{(\text {red })}\left(\exp \left(-\beta \hat{\mathcal{H}}_{\mathrm{p}}^{(0)}\right)\right)
$$

where the operation $\operatorname{Sp}_{(\text {red })}(\ldots)$ means the trace taken over the above selected states $|(k ; \alpha(k))\rangle \in$ $L_{2}(\Lambda ; \mathbb{C}) \otimes \Phi(\Lambda ; \mathbb{C})$.

This problem, being important for suitable applications of the polaron model under regard, is planned to be treated in detail in a work under preparation.

\section{Acknowledgements}

The authors are greatful to their colleagues from the ICTP Center in Trieste, Italy, for valuable discussions of the related physical aspects concerning the polaron model approximations. Authors cordially appreciate the Referee, whose remarks and suggestions proved so instrumental when preparing the manuscript, Prof. Z. Popowicz (Wroclaw University, Poland) and Prof. Denis L. Blackmore (NJIT, Newark NJ, USA) for very useful comments and discussions.

\section{References}

1. Bogolubov N.N., Bogolubov N.N. (jr.), Polaron Theory. Gordon and Breach Sci. Relativistic quantum fields. Mc Graw-Hill Book Co., NY, 1965.

2. Landau L.D., Z. Phys., 1933, 3, 664.

3. Bogolubov N.N., Plechko V.N., Teor. Mat. Fizika, 1985, 65, No. 3, 423-434

4. Bogolubov N.N., Ukr. Math. J., 1950, 2, No. 2.

5. Lee T.D., Low F.E., Pines D., Phys. Rev., 1953, 90, 297.

6. Brosens F., Devreese J.T., Phys. Stat. Sol., 1988, 145(b), 517.

7. Alexandrov A.S., Krebs A.B., Sov. Phys. Usp., 1992, 35(5), 1-85.

8. Alexandrov A.S., Phys. Rev. B, 2000, 61, 12315-12327.

9. Firsov Yu., Kudinov E., Sov. Fizika Tverd. Tela, 1997, 39, 215.

10. Reilly P.D., Harris R.A., Whaley K.B., Chem. Phys., 1991, 95, 8599.

11. Reilly P.D., Harris R.A., Whaley K.B., Chem. Phys., 1991, 97, 6975.

12. Dirac P.A.M., The Principles of Quantum Mechanics. Second Edition. Oxford, Clarendon Press, 1935.

13. Feynman R.P., Statistical Mechanics. W.A. Benjamin, 1972.

14. Ishihara A., Statistical Physics. Academic Press, New York, 1971.

15. Bogolubov N.N. (Jr.), Prykarpatsky A.K., Phys. Part. Nuclei, 1986, 17, No. 4, 789-827.

16. Mitropolsky Yu.A., Bogolubov N.N. (jr.), Prykarpatsky A.K., Samoylenko V.H., Integrable Dynamical Systems. Naukova Dumka Publ., Kyiv, 1987 (in Russian).

17. Bogolubov N.N, Bogolubov N.N. (jr.), Introduction Into Quantum Statistical Mechanics, Second Edition. World Scientific, 2009.

18. Raimes S., Many-Electron Theory. North-Holland Publ., 1972.

19. Petrina D.Ya., Mathematical Foundations of Quantum Statistical Mechanics. Academy of Sciences of Ukraine, Institute of Mathematics Publ., Kyiv, 1995 (in Russian). 
20. Doria M.M., Menikoff R., Sharp D.H., Phys. Rev. A, 1988, 37, No. 7, 2605-2607.

21. Lieb E.H., Thomas L.E., Commun. Math. Phys., 1983, 183, 11-519.

22. Lieb E.H., Seiringer R., Rep. Math. Phys., 2007, 59, No. 3, 389-399.

23. Kochetov E.A., Kuleshov S.P., Smondyrev M.A., Fiz. Elem. Chastits Atom. Yadra, 1982, 13, 635.

24. Rodriges C., Fedyanin V.K., Fiz. Elem. Chastits Atom. Yadra, 1984, 15, 870.

\title{
Представлення Боголюбова для поляронної моделі та їі повністю інтегроване наближення хаотичних фаз
}

\author{
М.М. Боголюбов (мол. $\sqrt{112}^{\sqrt[12]{2}}$ Я.А. Прикарпатський $\sqrt{344}$, А.А. Казарян \\ ${ }^{1}$ Математичний інститут ім. Стєклова Російської академії наук, Москва, Російська Федерація \\ 2 Міжнародний центр теоретичної фізики ім. Абдус Салама, Трієст, Італія \\ 3 Математичний інститут, Краківський педагогічний університет, Польща \\ 4 Дрогобицький педагогічний університет ім. Івана Франка, Львівська область, Україна \\ 5 Московський державний університет ім. Ломоносова, фізичний факультет, Москва, Російська \\ Федерація
}

Досліджується модель полярона в іонному кристалі в представленні Боголюбова, використовуючи особливе наближення хаотичних фаз. Виводиться та детально описується нова точно розв'язна наближена модель полярона. Аналітично отримано вільну енергію такої моделі при ненульовій температурі. Також обговорюється вільна енергія полярона в постійному магнітному полі при ненульовій температурі. На основі унітарного перетворення Боголюбова для поляронного гамільтоніана отримано дуже важливий новий результат: повна модель полярона є точно розв'язною.

Ключові слова: полярон, модель полярона, постійне магнітне поле, наближення хаотичних фаз 